\title{
How well did I do? The effect of feedback on affective commitment in the context of microwork
}

\author{
Anna-Maria Staiger \\ Technische Universität \\ Braunschweig \\ a.staiger@tu-braunschweig.de
}

\author{
Johannes Schmidt \\ Technische Universität \\ Braunschweig \\ johannes.schmidt@tu- \\ braunschweig.de
}

\author{
Dietrich von der Oelsnitz \\ Technische Universität \\ Braunschweig \\ d.oelsnitz@tu-braunschweig.de
}

\begin{abstract}
Crowdwork is a relatively new form of platformmediated and paid online work that creates different types of relationships between all parties involved. This paper focuses on the crowdworker-requester relationship and investigates how the option of receiving feedback impacts the affective commitment of microworkers. An online vignette experiment $(N=145)$ on a German crowdworking platform was conducted. We found that the integration of feedback options within the task description influences the affective commitment positively toward the requester as well as the perceived requester attractiveness.
\end{abstract}

\section{Introduction}

The increasing application of information technologies within the working environment leads to significant economic and organizational changes [1]. Organizational forms become more pluralistic and diverse $[2,3]$. New forms of work, such as crowdwork or online freelancing, evolve. Crowdwork, understood as a platform-mediated and paid online activity, bears challenges for all parties involved.

Requesters must mostly contend with an unknown crowd and possibly work results, which may be of low quality. Although work results can be rejected and payments can be retained by requesters [4], low-quality work is related to additional effort. In this sense, it seems important to avoid low-quality work results in advance. We assume that requesters are responsible for maintaining crowdworker performance by considering crowdworkers' (psychological) needs and expectations [5].

Although crowdworkers are not integrated directly within an organization and one might argue that any investment in a crowdworker-requester relationship is unnecessary, because crowdworkers do not miss social attachment or leadership at all, we presume HRM practices and leadership remain important within the given context [6-8]. Accordingly, we aim to show that phenomena such as organizational identification, engagement and commitment must be examined within environments of new, particularly digital or nonstandard forms of work [9-13].

The paper assumes that social bonds and especially organizational commitment (OC) are important aspects of performance and the crowdwork experience. Our research focuses on the relationship between the requester and the crowdworker, as well as the perceived $\mathrm{OC}$ in a microwork environment. We raise the question of whether the stimulation of the crowdworker-requester relationship by integrating the option of receiving feedback affects the perceived affective commitment (AC) toward a requester. To analyze the $\mathrm{AC}$, we conducted an experimental vignette study, where we manipulate the option of receiving feedback from the requester. To our best knowledge, our study addresses a gap within the literature and aims to provide a better understanding of the overall crowdwork experience with regard to perceived relationships.

The article is structured as follows. First, we provide an overview of the crowdwork environment. Second, we describe OC and its effects in traditional organizational settings. Furthermore, we review the literature concerning $\mathrm{OC}$ in the context of crowdwork and develop our hypotheses. We then describe our research design. After that, we present and discuss our findings. Finally, we conclude with a reflection on our contributions and discuss the limitations of our study. 


\section{Crowdwork and the nature of microwork}

\subsection{Definition of crowdwork}

Crowdwork can be seen as a new form of digital work arrangement within the so-called Gig-Economy [14].

Crowdwork $^{1}$ is based on the principles of crowdsourcing and can be understood as a paid ${ }^{2}$ and interactive collaboration between requesters (any profit or nonprofit organization, individual, group) and an undefined mass of individuals (crowd) [17]. The crowd consists of normally anonymous crowdworkers, who are characterized by different motivation levels, qualifications, skills and professional abilities $[15,18]$. Collaboration is initiated and coordinated through an open call on IT-mediated platforms [19].

There are diverse reasons for crowdworkers to participate in crowdworking activities. In addition to reasons such as fun, entertainment and the feeling of affiliation with a certain community [20-22], financial compensation is the most influential factor [20, 23, 24]. Therefore, crowdwork can be a considerable part of income. It offers the possibility to engage in crowdwork full-time, part-time or as an additional job [25-28].

The literature shows that crowdwork differs from other forms of platform-mediated work, such as 'workon-demand via app' or 'gig-work'. The latter implies work activities, which are assigned through online platforms or mobile apps, but in contrast to crowdwork, the final execution of gig work is locally bound (e.g., transport and delivery services ${ }^{3}$ ) [14].

In this paper, we focus on crowdwork as a type of paid and platform-mediated digital work based on crowdsourcing principles. Furthermore, crowdwork is characterized by local distance, anonymity and autonomy [29]. Accordingly, all forms of nonremunerated, contest-based and locally bound crowdsourced activities are excluded from our definition.

1 Within the literature, "Gig-Work" or "Crowdwork" are often used synonymously.

2 Different from other forms of crowd- or gig- activities, i.e., citizen science or voluntary participation in online communities such as Wikipedia or human-based computation games $[15,16]$, crowdwork is characterized as gainful employment, which implies that participating crowdworkers are paid and remunerated for their engagement.

3 Typical locally bound tasks are conducted on platforms such as Uber, Deliveroo and MyHammer.

\subsection{Characteristics of Microwork}

Crowdwork enables requesters to outsource various tasks without establishing a long working relationship [13]. Hence, crowdworkers operate mainly as self-employed agents with a high degree of autonomy and flexibility [20,25]. There are basically two types of crowdwork: microwork and online freelancing [30].

Microwork, also referred to as 'cognitive piecework' [31] or 'human intelligence tasks' [20], is characterized by a high degree of granularity, which means that larger tasks are decomposed into discrete small units, which are simple and easy to perform. [32]. Although cognitive piecework or microtasks do not require distinct qualifications, there remains a need for human intelligence because tasks cannot be fully automated yet [33]. Examples of typical microwork tasks are tagging pictures, participating in surveys, writing brief product descriptions or transcribing audio data $^{4}$ [34]. Because of the simplicity of this type of work, crowdworkers normally gain just micro payments for each task they complete. There is no hourly wage $[31,35,36]$. Taking this fact into consideration, microwork may create precarious working conditions [36-38].

In addition to microwork, online freelancing is another type of online crowdwork. It follows the principles of crowdwork, but it requires a higher level of expertise and professional skills. Examples of online freelancing are web design, software programming or graphic design ${ }^{5}[30]$.

We focus on microtask crowdwork and corresponding platforms. We use the term crowdwork synonymously to microwork. Furthermore, we understand requesters as organizations. This understanding seems appropriate because we conceptualize crowdwork as an at least partly interactive collaboration between organizations and external crowdworkers.

\subsection{Context and research question}

The overall research concerning crowdwork and platform-mediated work has gained greater attention in recent years [39]. Prior research focuses, for example, on demographics and crowdworkers' personal life contexts [40, 41] or platform design and platform policies and governance systems $[42,43]$.

4 Typical microwork platforms include, for example, MTurk, Clickworker, AppJobber and CrowdFlower.

5 Typical online freelancing platforms include, for example,

Upwork, Jovoto and 99designs. 
Another stream of research analyzes motives for participating in crowdwork and the overall perceptions workers develop within the crowdwork experience [5, $20,27,44,45]$. For crowdworkers, experience is shaped by perceived information asymmetry and an imbalance of power [31, 37, 42, 48].

With an increasing application of crowdwork, it is necessary to develop a deeper understanding of the crowdwork environment and the triangular relationship between crowdworkers, requesters and platform providers.

For requesters, two main questions seem especially important: (1) how to secure task quality and (2) how to motivate workers to try their best [46, 47].

Martin et al. (2014) show that despite the lack of direct interaction with the requester, the emotional involvement of crowdworkers can be measured [49]. Hence, it is clear that the short-term relationship between the requester and the crowdworker seems to be more considerable than previously assumed. The crowdworking process addresses deeper individual motives, and crowdworkers strive for social contact with the requester, although the microwork environment does not directly encourage social attachment. Consequently, it can be assumed that these insights about relationships might be relevant for addressing the two questions posed above.

Factors for establishing a relationship between the requester and the crowdworker can be identified in the integration of communication and feedback practices $[49,50]$. Direct feedback can be considered positive for improving crowdworkers' accuracy and task completion time [51]. Building on these insights from the literature and our assumption of a triangular relationship between all parties involved, we argue that investing in a relationship between the requester and the crowdworker may have a positive impact on the individual crowdwork experience. Consequently, the following research question is posed: What type of effects result from the investment in a more specific relationship between the requester and the crowdworker?

\section{Commitment in the Context of Crowdwork}

\subsection{Organizational Commitment}

Organizational commitment (OC) is a widespread concept that describes and analyzes the relationship between employees and employers and addresses the overall attachment, involvement and identification with an organization. OC measures the degree to which an employee accepts organizational values and objectives and hence builds loyalty in relation to an employer or organization $[52,53]$. OC helps to create a better understanding of employee behavior within organizations and can be interpreted as an attitude of employees toward an organization [54, 55]. One framework that addresses different dimensions of OC is the Three Component Model proposed by Meyer and Allen (1991) (see Table 1) [56].

\section{Table 1. Components of Organizational Commitment}

\begin{tabular}{|l|l|}
\hline $\begin{array}{l}\text { Affective } \\
\text { Commitment } \\
\text { (AC) }\end{array}$ & $\begin{array}{l}\text { Desire to stay in the } \\
\text { organization; emotional } \\
\text { attachment }\end{array}$ \\
\hline Continuance & Cost-orientated need to stay in \\
Commitment & $\begin{array}{l}\text { an organization; rational } \\
\text { attachment }\end{array}$ \\
\hline NC) & $\begin{array}{l}\text { Moral duty or perceived } \\
\text { obligation to stay in an } \\
\text { Commitment } \\
\text { (NC) }\end{array}$ \\
\hline
\end{tabular}

Allen and Meyer (1991) note that employees can experience all three components of OC simultaneously, whereas the general degree of an employee's experience varies [56]. In this study, we understand OC as an overall individual attitude that affects the performance-based behavior of crowdworkers and helps to explain the willingness to work for a requester.

\subsection{OC in the context of Crowdwork and Research Hypotheses}

The preceding description of crowdwork indicates similarities to other ICT-based or less place-bound ondemand types of employment: that is, crowdwork is performed with the support of ICT outside a requester's organization. Accordingly, there is no or less contact with other employees and clients. Therefore, work is performed mainly in isolation [57, 58]. The relationship to the requester is essentially characterized by a limited temporal and functional connection [59, 60]. Nevertheless, as human beings, crowdworkers must create a professional identity and should receive recognition for their work to stay motivated and to fulfill their basic psychological needs [61]. Moreover, it can be argued that crowdworkers, as external working partners, strive for social relationships to perceive, for example, meaningfulness and affiliation. Therefore, it is important to gain additional insight into individual crowdwork experiences and to examine phenomena such as OC within the crowdwork environment.

While researchers have shown that OC can be cultivated in similar types of work, such as contract 
work and on-demand work [9, 62, 63], knowledge concerning crowdwork remains limited. There is little research on $\mathrm{OC}$ to the requester. Our research therefore aims to provide more insight into this type of relationship within crowdworking.

First, existing crowdworking literature regarding OC focuses mainly on the relationship between crowdworkers and platforms. By signing up on a crowdworking platform as a free agent, a formal relationship between the crowdworker and the platform is constituted, although it cannot be evaluated as a traditional organizational employer-employee relationship [64].

In microwork environments, platforms shape digital working conditions and strongly affect the crowdwork experience $[57,58]$. Thus, there is initially no direct relationship to the requester $[15,38]$. Crowdworkers can experience the platform as a virtual organization instead and can develop a feeling of belongingness toward the platform. Different studies underline that crowdworkers strive and aim for longerterm relationships with a platform because of a missing employer [37].

Knowing that crowdworkers can be active on several platforms simultaneously and are consequently able to exert bargaining power, crowdwork platforms themselves are interested in affecting the engagement and commitment of crowdworkers toward the platform. For these reasons, platforms use, among others, the integration of crowdwork communities as a central management strategy that serves as a substitute for social bonds $[37,58]$. With communities and tools for communication with other crowdworkers, platforms stimulate the basic need for relatedness, which creates considerable potential for building up identification with the platform as well as with the microwork community [37, 65]. In this context, Ihl et al. (2020) underline that microwork communities function as alternative sources of social support and can enhance crowdworkers' identification and engagement [66]. Schulten \& Schäfer (2015) explain that creating a community within crowdsourced cocreation projects can be viewed as a central determinant of AC [67]. In general, the literature shows that the promotion of communities within the crowdwork environment helps to stimulate OC toward the platform.

Second, a review of the literature indicates that establishing a relationship between the crowdworker and the requester within the microwork environment is rather limited [37]. One reason might be the potentially missing necessity for investing in such relationships as long intermediaries manage the crowdworking process. Nevertheless, Troll et al. (2019) elaborate that emotional bonds can be developed toward the requester despite integrating a platform [68].
Wang et al. (2020) note that the willingness to engage in crowdworking and receive acknowledgment for working is affected by positive or negative experiences, characterized by perceived demands and resources $[69,70]$.

Therefore, job demands (e.g., work pressure, cognitive demands) and job resources (e.g., feedback, requester support) have an effect on perceived (platform) commitment [69]. It can be assumed that crowdworkers react positively to mechanisms and instruments, such as feedback from requesters, requester support and interaction possibilities, which are similar to management practices in traditional organizational settings. Certainly, social support from requesters, such as receiving advice or appreciation, is limited [20, 57]. In microwork settings, feedback often persists in the transfer of payments, i.e., money is associated with positive feedback [57].

We propose that in the context of dehumanizing work conditions [15], there are deficits in submitting and integrating requester feedback, as feedback is one descending characteristic of a good job design, which can result in high job satisfaction, job motivation and OC $[71,72]$. We argue that the possibility of interacting with the requester may enhance the $\mathrm{OC}$ of crowdworkers to the requester because receiving feedback and the perception of social support are positively related to $\mathrm{OC}[73,74]$. Consequently, we expect that if workers are committed to the requester, they may have greater interest in ensuring good-quality results. Based on the literature and suitable interaction practices between both parties apart from the platform, we assume feedback to be important for shaping the crowdworker-requester relationship and consequently for affecting OC.

In this article, OC is understood as crowdworkers' psychological and emotional attachment to a client organization respectively requester. High OC scores may predict better job quality and performance rates, which result in noticeable organizational benefits.

Although it can be argued that the platform can be perceived as an employer or organization to which the crowdworkers are affiliated [37], we aim to show that there is also potential for building up OC to the requester.

Within our study, we focused on the affective component of OC. One reason for this decision is the general discussion of the microwork environment. The use of CC and NC seems debatable when studying the relationship between the requester and the crowdworker within the triangular setting of crowdwork. NC addresses the obligation to work for an organization because one feels that doing so is the 'right thing to do'. According to the character of microwork, we do not see this moral obligation as a 
suitable component within our study. CC addresses the perceived need to stay in an organization because the loss one would experience by leaving an organization is greater than the benefit one might gain. In our view, $\mathrm{CC}$ is rather relevant when studying the relationships of crowdworkers and platforms, as ranking systems and gained experiences have a noticeable impact, for example, on the distribution of tasks [58]. For this reason, we assume $\mathrm{AC}$ to be the most adequate component to study commitment in the presumed emotional crowdworker-requester relationship.

Requesters must contend with the question of how to motivate workers to try their best. Another question that appears in this context is how to attract crowdworkers to accept tasks from individual requesters. The literature demonstrates that money and the amount of remuneration are the most impactful factors in microwork environments when deciding to accept a specific task [18, 57]. Further important aspects can be identified in the creation of meaningfulness and the task itself [5]. We also argue that crowdworkers tend to take on specific tasks when they perceive the requester as fair regarding payment behavior and related explanations by occurring rejections [75]. Therefore, we think the option of feedback may also have an impact on the willingness to work for a certain requester. Hence, we subsequently expect that the option for feedback affects the perceived requester attractiveness.

Based on the above arguments, we formulate the following hypotheses:

H1: The option of receiving feedback from the requester affects the perceived organizational affective commitment.

H2: The option of receiving feedback from the requester affects the perceived requester attractiveness.

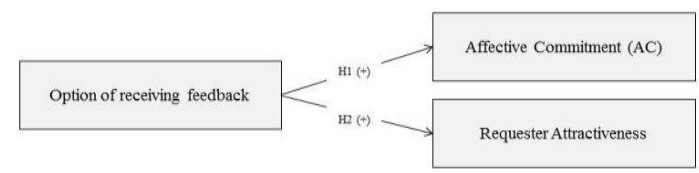

Figure 1. Research Model

\section{Research Method}

\subsection{Study design}

To address our research hypotheses, we conducted an online vignette experiment, also known as the 'factorial survey approach' [76]. This method combines the advantages of traditional lab experiments and survey methods [77]. A vignette is a carefully constructed brief description of a person or a situation that contains information, which is presented to respondents to obtain a judgment about that person or situation [78]. Within this description, the independent variable(s) can be systematically varied, which is a crucial aspect of making causal inferences [79].

In our design, vignettes were used in an online survey to describe a job that typically occurs on microworking platforms ${ }^{6}$. To increase ecological validity and to expose participants to an environment that feels natural to them, the vignette was designed using real-life job descriptions on crowdworking platforms [80].

The first part of the vignette included basic facts about the requester and the task description. Then, we varied the factor of interest (= option of receiving feedback from the requester [yes/no]). At the end of the task description, participants in the treatment group (= feedback [yes]) were given the following information: 'After completion of the task, we are happy to offer you the opportunity to receive personal feedback on your work. Because we regularly add new products to our portfolio, good cooperation is important to $u s^{\prime}$. In contrast, the participants in the control group (= feedback [no]) only received basic information about the requester and the task description. To reduce the chance of social desirability bias and to avoid cognitive overload among respondents, we used a between-subject design in which each respondent was randomly assigned to the treatment group or the control group [78, 81].

\subsection{Measurement}

The two dependent variables AC and organizational attractiveness were measured with a Likert scale that ranged from 1 (strongly disagree) to 5 (strongly agree). The five items that measured AC (e.g., 'I am proud to work for this requester') were taken from Felfe et al. (2002) [82]. The four items for organizational attractiveness (e.g., 'I find this a very attractive requester to work for') were taken from Aiman-Smith et al. (2001) [83]. The items used were adapted to the study context. The Cronbach's alpha coefficient for the AC scale was 0.88, and that for the organizational attractiveness scale was 0.91 , indicating good internal consistency.

We conducted attention checks to verify whether the participants had read the case scenario diligently and with sufficient attention [84]. In line with other experimental studies [85], all participants who did not answer the questions correctly were excluded from the

6 Full vignettes are available from the authors upon request. 
following analysis. Furthermore, the effectiveness of vignettes depends on how realistic and plausible the research participant perceived the vignette content [86]. Therefore, we asked the participants to indicate whether they were able to put themselves into the presented scenario (from $1=$ not at all easy to $5=$ very easy) and whether they thought the task was realistic (from $1=$ not at all realistic to $5=$ very realistic). We excluded participants who could not or could only poorly put themselves in the presented situation (scale $<3$ ) and who rated the scenario as not or only slightly realistic $($ scale $<3)$.

\section{Research Results}

\subsection{Sample characteristics}

To recruit participants for our experiment, we initiated a remunerated job (microtask) on a German crowdworking platform (namely, 'clickworker.de') in December 2020. After exclusion due to attention check failure and related assumptions, our final sample consisted of 145 real crowdworkers, of which 44 percent were female and 56 percent were male. Participants' age varied between 18 and 71 years $(\mathrm{M}=$ 38.66 years; $\mathrm{SD}=13.12$ ).

Given these demographics, our sample is representative of other related studies within the microwork environment [87]. As noted above, participants were randomly assigned to the treatment (n $=78)$ or control group $(n=67)$.

\subsection{Testing the hypotheses}

A one-way MANOVA was conducted to investigate whether there were significant differences in the mean scores of the two dependent variables across the two conditions.

There were no outliers in the data. Correlations between dependent variables were low $(r<.90$; [88]), indicating that multicollinearity was not a confounding factor in the analysis. There was homogeneity of the error variances, as assessed by Levene's test $(p>.05)$, and homogeneity of covariances, as assessed by Box's test $(p>.001)$.

A one-way MANOVA showed a statistically significant difference between the two factor levels of the independent variable on the combined dependent variables, $\mathrm{F}(2,142)=6.270, \mathrm{p}<.001$, partial $\eta^{2}=.081$, Wilk's $\Lambda=.921$, thus providing support for our hypotheses. Specifically, respondents expressed higher OC and higher organizational attractiveness when the option of receiving feedback from the requester after completing the task was given (for OC: MFeedback[yes] =
3,4 vs. MFeedback[no] $=2,9 ;$ for organizational attractiveness: MFeedback[yes] = 3,8 vs. MFeedback[no] =3,5).

\section{Discussion and Implications}

In this paper, we examined the influence of feedback on the perceived AC toward a requester as well as the perceived requester attractiveness. First, we found that the option of receiving feedback from the requester has a significant positive effect on the perceived AC (H1), although the option of perceiving feedback is only mentioned in the task description. Second, the results show that crowdworkers significantly associate the requester as an attractive client when feedback is optionally integrated (H2). Accordingly, the results confirm the assumption that investment in a more specific relationship to crowdworkers could have positive side effects.

To our best knowledge, our study is one of the first empirical examinations to adopt an experimental approach to analyze the established psychological construct OC within the crowdworker-requester relationship. Our results reflect that the option for communication and the chance of acknowledgment from the requester seem to be relevant even in the detached microwork environment. Regarding individual social and psychological needs, recent studies focus mainly on online freelancing or creative design tasks, where feedback and communication with the requester is an inherent part of the task [89, 90]. The necessity for addressing such individual needs has hardly been considered in microwork contexts, possibly because of the granularity of tasks, which may prevent the development of, for instance, OC. Nevertheless, the option of receiving feedback from the requester might help to build up a quasi-long-term relationship because the anonymity of both parties disappears. Consequently, crowdworkers are able to search for requesters with whom they have previously had good experiences.

Some managerial implications arise: the positive effects of OC should be further considered. The empirical literature on $\mathrm{OC}$ discusses positive relations of OC with performance, motivation and worker wellbeing [73, 91, 92]. Although microwork is not yet a fully implemented alternative to traditional work, requesters can likely benefit from crowdworkers, who are emotionally committed $[37,58]$. It can be argued that requesters may have lower rejection rates and can subsequently reinforce the cost and time advantages of crowdwork. Additionally, requesters may benefit from their attributed attractiveness to develop their own 'requester brand'. As crowdworking gains increasing interest, crowdworkers can exert their bargaining power and decide which platform they prefer and 
whether they work for one requester or another. This choice may potentially lead to a "war for crowdworkers", which highlights the relevance of considering requester attractiveness.

Our study also has valuable theoretical implications and aspects for future research. First, we provide evidence on the positive impact and effects of the option for receiving feedback from the requesters in a microwork environment. Second, we apply and empirically assess the construct of OC within the crowdworker-requester relationship. Our results reveal that microworkers strive to fulfill basic psychological needs, which is consistent with traditional organizational literature. Third, we attempted to gain more insight into drivers and determinants, which could affect crowdworkers' behavior. Our results confirm that feedback might be an important aspect of understanding and directing crowdworkers within microwork environments.

Nevertheless, our study has some limitations. A (hypothetical) situation described in a vignette can never be completely realistic and may also be susceptible to individual misperceptions or misreactions. Therefore, the transferability of our study results might be limited, even though we have attempted to design our vignettes based on real-life jobs on existing crowdworking platforms. In addition, there are some limitations of experimental betweensubject designs in regard to perceptions and situational judgments, as is the case with factorial surveys. Accordingly, between-subject designs are (contrary to within-subject designs) associated with measurement problems [93]. However, we argue that the results of a within-subject design would have been biased in the present context because participants would not have been blind to the conditions, thus resulting in memory and sequence effects.

Our results may offer guidance for future research. We recommend replicating certain microwork studies while examining other dependent variables, including for instance task performance, task quality, engagement and other suitable constructs. Regarding $\mathrm{OC}$, there is a need for a deeper understanding of NC and $\mathrm{CC}$. We propose qualitative studies to gain a deeper understanding of the individual crowdwork experience. Furthermore, it would be insightful to manipulate the content and/or format of the requester's feedback to crowdworkers in further experiments. It will also be important to extend the generalizability of this study by examining whether the option for receiving feedback may have a similar effect on crowdworkers on other platforms and/or in other contexts.

Finally, our study indicates that investing in social relationships within the microwork environment should not be underestimated by either platforms or requesters. Requesters should expect microworkers to be external working partners who also strive for meaning, affiliation and recognition.

\section{References}

[1] M. Kenney, P. Rouvinen, T. Seppälä, and J. Zysman, "Platforms and industrial change", Industry and Innovation, 26(8), 2019, pp. 871-879.

[2] A.L. Kallenberg, "Rethinking the sociology of Work, Workers and the Workplace", Labour \& Industry: a journal of the social and economic relations of work, 19(3), 2009, pp. 29-48.

[3] G.M. Spreitzer, L. Cameron, and L. Garrett, "Alternative Work Arrangements: Two Images of the New World of Work", Annual Review of Organizational Psychology and Organizational Behavior, 4(1), 2017, pp. 473-499.

[4] M. Buhrmester, T. Kwang, and S.D. Gosling, “Amazon's Mechanical Turk: A New Source of Inexpensive, Yet High-Quality, Data?", Perspectives on Psychological Science, 6(1), 2011, pp. 3-5.

[5] D. Kost, C. Fieseler, and S.I. Wong, "Finding meaning in a hopeless place? The construction of meaningfulness in digital microwork", Computers in Human Behavior, 82, 2018, pp. 101-110.

[6] D. Cross and J. Swart, "The (ir)relevance of human resource management in independent work: Challenging assumptions", Human Resource Management Journal, 2021, pp. 1-15.

[7] J. Duggan. U. Sherman, R. Carbery, and A.J. McDonnell, "Algorithmic management and app-work in the gig economy: A research agenda for employment relations and HRM", Human Resource Management Journal, 2020(30), 2019, pp. 114-132.

[8] J.T. Bush, and R.M. Balven, "Catering to the crowd: An HRM perspective on crowd worker engagement", Human Resource Management Review, 31(1), 2021, pp. 1-16.

[9] E. George, and P. Chattopadhyay, "Non-standard work an workers: Organizational implications", Conditions of Work and Employment Series, 2016.

[10] R.E. Frieder, "The Rules of Social Exchange: Unchanged but More Important Than Ever", Industrial and Organizational Psychology, 11(3), 2018, pp. 535541.

[11] Y.G.T. van Rossenberg, H.J. Klein, K. Asplund, K. Bentein, et al., "The future of workplace commitment: key questions and directions", European Journal of Work and Organizational Psychology, 27(2), 2018, pp. $153-167$.

[12] T. McKeown, and R. Cochrane, "Independent professionals and the potential for HRM innovation", Personnel Review, 46(7), 2017, pp. 1414-1433.

[13] K.M. Kuhn, "The Rise of the "Gig Economy" and Implications for Understanding Work and Workers", Industrial and Organizational Psychology, 9(1), 2016, pp. $157-162$.

[14] V. De Stefano, "The Rise of the "Just-in Time Workforce": On-Demand Work, Crowd Work and 
Labour Protection in the "Gig-Economy"”, Comparative Labor Law \& Policy Journal, 2015.

[15] A. Kittur, J.V. Nickerson, M. Bernstein, E. Gerber, et al., "The future of crowd work", in Proceedings of the 2013 conference on Computer supported cooperative work, pp. 1301-1317.

[16] L. Von Ahn, and L. Dabbish, "Labeling images with a computer game", in Proceedings of the SIGCHI Conference on Human Factors in Computing Systems, 4/24/2004 - 4/29/2004. 2004. ACM: New York, NY, pp. 319-326.

[17] I. Blohm, S. Zogaj, U. Bretschneider, and J.M. Leimeister, "How to Manage Crowdsourcing Platforms Effectively?", California Management Review, 60(2), 2018, pp. 122-149.

[18] R. Brewer, M.R. Morris, and A.M. Piper, "Why would anybody do this?", in \#chi4good: CHI 2016: San Jose, CA, USA, May 7-12 : proceedings : the 34th Annual CHI Conference on Human Factors in Computing Systems, pp. 2246-2257.

[19] J. Howe, "The Rise of Crowdsourcing", Wired Magazine, 14.06, 2006.

[20] X. Deng, and K.D. Joshi, "Why Individuals Participate in Micro-task Crowdsourcing Work Environment: Revealing Crowdworkers' Perceptions", Journal of the Association for Information Systems, 17(10), 2016, pp. 649-673.

[21] M. Hossain, 'Users' motivation to participate in online crowdsourcing platforms", in 2012 International Conference on Innovation, Management and Technology Research (ICIMTR 2012): Malacca, Malaysia, 21 - 22 May 2012, pp. 310-315.

[22] D.C Brabham, "Crowdsourcing as a Model for Problem Solving", Convergence: The International Journal of Research into New Media Technologies, 14(1), 2008, pp. 75-90.

[23] N. Kaufmann, T. Schulze, and D. Veit, "More than fun and money. Worker Motivation in Crowdsourcing - A Study on Mechanical Turk", 2011, in AMCIS 2011 Proceedings.

[24] Ipeirotis,P. Demographics of Mechanical Turk, 2010.

[25] D. Durward, I. Blohm and J.M. Leimeister, "The Nature of Crowd Work and its Effects on Individuals' Work Perception", Journal of Management Information Systems, 37(1), 2020, pp. 66-95.

[26] A.M. Brawley, "The Big, Gig Picture: We Can't Assume the Same Constructs Matter", Industrial and Organizational Psychology, 10 (4), 2017, pp. 687-696.

[27] A.M. Brawley, and C.L.S. Pury, "Work experiences on MTurk: Job satisfaction, turnover, and information sharing", Computers in Human Behavior, 54, 2016, pp. 531-546.

[28] J. Ross, L. Irani, M.S. Silberman, A. Zaldivar, and B. Tomlinson, "Who are the crowdworkers?: shifting demographics in mechanical turk", in CHI '10 Extended Abstracts on Human Factors in Computing Systems, 4/10/2010 - 4/15/2010. 2010. ACM: New York, NY, pp. 2863-2872.

[29] S.J. Ashford, B.B. Caza, and E.M. Reid, "From surviving to thriving in the gig economy: A research agenda for individuals in the new world of work",
Research in Organizational Behavior, 38, 2018, pp. 2341.

[30] S.C. Kuek, C. Paradi-Guilford, T. Fayomi, S. Imaizumi, and P. Ipeirotis, "The Global Opportunity in online Outsourcing", 2015.

[31] A. Felstiner, "Working the Crowd: Employment and Labor Law in the Crowdsourcing Industry", Berkeley Journal of Employment and Labor Law, 31(1), 2011, pp. 143-203.

[32] T. Hoßfeld, M. Hirth, and P. Tran-Gia, "Modeling of Crowdsourcing Platforms and Granularity of Work Organization in Future Internet", 2011, pp. 142-149.

[33] E. Kaganer, E. Carmel, R. Hirschheim, and T. Olsen, "Managing theHuman Cloud", MIT Sloan Management Review, 54(2), 2013, pp. 23-32.

[34] U. Gadiraju, R. Kawase, and S. Dietze, "A Taxonomy of Microtasks on the Web", 25th ACM Conference on Hypertext and Social Media (HT'14), 2014, pp. 218223.

[35] J. Berg, "Income security in the on-demand economy: Findings and policy lessons from a survey of crowdworkers", Conditions of Work and Employment Series(74), 2016.

[36] B. Bergvall-Kåreborn, and D. Howcroft, "Amazon Mechanical Turk and the commodification of labour", New Technology, Work and Employment, 29(3), 2014, pp. 213-223.

[37] N. Panteli, A. Rapti, and D. Scholarios, "If He Just Knew Who We Were': Microworkers' Emerging Bonds of Attachment in a Fragmented Employment Relationship", Work, Employment and Society, 34(3), 2020, pp. 476-494.

[38] J. Prassl, and M. Risak, "Uber, Taskrabbit, \& Co: Platforms as employers? Rethinking the legal analysis of Crowdwork", Comparative Labour Law and Policy Journal, Oxford Legal Studies Research Paper No. 8, 2016, pp. 1-30.

[39] A. Margaryan, and H. Hofmeister, "The Life Course: An interdisciplinary framework for broadening the scope of research on crowdwork", Human Computation, 8, 2021, pp. 43-75.

[40] V. Lehdonvirta, "Flexibility in the gig economy: managing time on three online piecework platforms", New Technology, Work and Employment, 33(1), 2018, pp. 13-29.

[41] D. Difallah, E. Filatova, and P. Ipeirotis, "Demographics and Dynamics of Mechanical Turk Workers", in WSDM'18: Proceedings of the Eleventh ACM International Conference on Web Search and Data Mining: February 5-9, 2018, pp. 135-143.

[42] M. Graham, I. Hjorth, and V. Lehdonvirta, "Digital labour and development: impacts of global digital labour platforms and the gig economy on worker livelihoods", Transfer (Brussels, Belgium), 23(2), 2017, pp. 135-162.

[43] D. Retelny, M.S. Bernstein, and M.A. Valentine, "No Workflow Can Ever Be Enough", Proceedings of the ACM on Human-Computer Interaction, 1 (CSCW), 2017, pp. 1-23. 
[44] A. Al-Ani, and S. Stumpp, "Rebalancing interests and power structures on crowdworking platforms", Internet Policy Review, 5(2), 2016, pp. 1-19.

[45] E. Bucher, C. Fieseler, and C. Lutz, "Mattering in digital labor", Journal of Managerial Psychology, 34(4), 2019, pp. 307-324.

[46] T. Straub, H. Gimpel, F. Teschner, and C. Weinhardt, "Feedback and performance in crowd work: a real effort experiment", Proceedings of the European Conference on Information Systems, 2014, pp. 1-10.

[47] J. Wang, P.G. Ipeirotis, and F. Provost, "Quality-Based Pricing for Crowdsourced Workers", NYU Working Paper No. 2451/31833, 2013.

[48] M.S. Silberman, J. Ross, L. Irani, and B. Tomlinson, "Sellers' problems in human computation markets", in Proceedings of the ACM SIGKDD Workshop on Human Computation, Washington DC, 7/25/2010 7/25/2010. 2010. ACM: New York, NY, pp. 18-21.

[49] D. Martin, B.V. Hanrahan, J. O`Neill, and N. Gupta, "Being A Turker", CSCW'14 17th ACM conference on Computer supported cooperative work \& social computing, 2014, pp. 224-235.

[50] U. Gadiraju, and G. Demartini, "Understanding Worker Moods and Reactions to Rejection in Crowdsourcing", 25th ACM Conference on Hypertext and Social Media (HT'14), 2019, pp. 211-220.

[51] U. Gadiraju, B. Fetahu, and R. Kawase, "Training Workers for Improving Performance in Crowdsourcing Microtasks", Proceedings of the 10th European Conference on Technology Enhanced Learning, 2015, pp. 100-114.

[52] D. van Knippenberg, and E. Sleebos, "Organizational identification versus organizational commitment: selfdefinition, social exchange, and job attitudes", Journal of Organizational Behavior, 27(5), 2006, pp. 571-584.

[53] C.J. Mottaz, "Determinants of Organizational Commitment", Human Relations, 41(6), 1988, pp. 467482.

[54] H.L. Angle, and J.L. Perry, "Organizational Commitment", Work and Occupations, 10(2), 1983, pp. 123-146.

[55] O.N. Solinger, W. van Olffen, and R.A. Roe, "Beyond the three-component model of organizational commitment", The Journal of applied psychology, 93(1), 2008, pp. 70-83.

[56] J.P. Meyer, and N.J. Allen, "A Three-Component Conceptualization of Organizational Commitment", Human Resource Management Review, 1(1), 1991, pp. 61-89.

[57] C. Fieseler, E. Bucher, and C.P. Hoffmann, "Unfairness by Design? The Perceived Fairness of Digital Labor on Crowdworking Platforms", Journal of Business Ethics, 156, 2019, pp. 987-1005.

[58] C. Gerber, and M. Krzywdzinski, "Brave New Digital Work? New Forms of Performance Control in Crowdwork", in Work and Labor in the Digital Age, S. P. Vallas, A. Kovalainen, Editor, 2019, pp. 121-143.

[59] K.W. Rockmann, and G.A. Ballinger, "Intrinsic motivation and organizational identification among ondemand workers", The Journal of applied psychology, 102(9), 2017, pp. 1305-1316.
[60] V. De Stefano, "Introduction: Crowdsourcing, the GigEconomy and the Law", Comparative Labor Law \& Policy Journal, 37(3), 2016.

[61] E.L. Deci, and R.M. Ryan, "Self-Determination Theory", in Theories of social psychology, P.A. van Lange, Editor. 2012, pp. 416-436.

[62] G. van Breugel, W. van Olffen, and R. Olie, "Temporary Liaisons: The Commitment of 'Temps' Towards Their Agencies", Journal of Management Studies, 42(3), 2005, pp. 539-566.

[63] S.J. Ashford, E. George, and R. Blatt, "2 Old Assumptions, New Work", The Academy of Management Annals, 1(1), 2007, pp. 65-117.

[64] K.M. Kuhn, and A. Maleki, "Micro-entrepreneurs, Dependent Contractors, and Instaserfs: Understanding Online Labor Platform Workforces", Academy of Management Perspectives, 31(3), 2017, pp. 183-200.

[65] M. Boons, D. Stam, and H.G. Barkema, "Feelings of Pride and Respect as Drivers of Ongoing Member Activity on Crowdsourcing Platforms", Journal of Management Studies, 52(6), 2015, pp. 717-741.

[66] A. Ihl, K. S. Strunk, and M. Fiedler, "The mediated effects of social support in professional online communities on crowdworker engagement in micro-task crowdworking", Computers in Human Behavior, 113, 2020, 106482, pp. 1-11.

[67] M.B. Schulten, and F. Schaefer, "Affective commitment and customer loyalty in crowdsourcing: antecedents, interdependencies, and practical implications", The International Review of Retail, Distribution and Consumer Research, 2015, pp. 516-528.

[68] J. Troll, I. Blohm, and J.M. Leimeister, "Why Incorporating a Platform-Intermediary can Increase Crowdsourcees' Engagement", Bus Inf Syst Eng, 61(4), 2019, pp. 433-450.

[69] Y. Wang, K. Papangelis, I. Lykourentzou, H.N. Liang, et al., "In Their Shoes: A Structured Analysis of Job Demands, Resources, Work Experiences, and Platform Commitment of Crowdworkers in China", PACM on Human-Computer Interaction, 4, 2020, pp. 1-40.

[70] E. Demerouti, A.B. Bakker, F. Nachreiner, and W.B. Schaufeli, "The job demands-resources model of burnout", The Journal of applied psychology, 86(3), 2001, pp. 499-512.

[71] J. Hackman, and G.R. Oldham, "Motivation through the design of work: test of a theory", Organizational Behavior and Human Performance, 16(2), 1976, pp. 250-279.

[72] J. Krasman, "Putting feedback-seeking into "context": job characteristics and feedback-seeking behaviour", Personnel Review, 42(1), 2012, pp. 50-66.

[73] A. Panaccio, and C. Vandenberghe, "Perceived organizational support, organizational commitment and psychological well-being: A longitudinal study", Journal of Vocational Behavior, 75(2), 2009, pp. 224-236.

[74] M. van Vuuren, M.D. de Jong, and E.R. Seydel, "Direct and indirect effects of supervisor communication on organizational commitment", Corporate Communications: An International Journal, 12(2), 2007, pp. 116-128. 
[75] P. Alpar, and L. Osterbrink, "Antecedents and Consequences of Perceived Fairness in Pay for Crowdwork", ECIS 2018.

[76] P.H. Rossi, and A.B. Anderson, "The factorial survey approach: an introduction", in Measuring social jugements: the factorial survey approach, P.H. Rossi and S.L. Nock, Editor. 1982. Sage: Beverley Hills, pp. 1567.

[77] I. Engelmann, "Vignette Research", in International Encyclopedia of Communication Research Methods, J. Matthes, C. Davis and R. Potter, Editor. 2017.

[78] C.S. Alexander and H.J. Becker, "The Use of Vignettes in Survey Research", Public Opinion Quarterly, 42(1), 1978, pp. 93-104.

[79] D.B. Rubin, "Comment: Which Ifs Have Causal Answers", Journal of the American Statistical Association, 81(396), 1986, pp. 961-962.

[80] H. Aguinis, and K.J. Bradley, "Best Practice Recommendations for Designing and Implementing Experimental Vignette Methodology Studies", Organizational Research Methods, 17(4), 2014, pp. 351-371.

[81] S. Walzenbach, "Hiding Sensitive Topics by Design? An Experiment on the Reduction of Social Desirability Bias in Factorial Surveys", Survey Research Methods, 13(1), 2019, pp. 103-121.

[82] J. Felfe, B. Six, R. Schmook, and C. Knorz, „Commitment Organisation, Beruf und Beschäftigungsform (COBB)“, Zusammenstellung sozialwissenschaftlicher Items und Skalen (ZIS), 2002.

[83] L. Aiman-Smith, T. N. Bauer, and D. M. Cable, "Are You Attracted? Do You Intend to Pursue? A Recruiting Policy-Capturing Study", Journal of Business and Psychology, 16(2), 2001, pp. 219-237.

[84] A.W. Meade, and S.B. Craig, "Identifying careless responses in survey data", Psychological Methods, 17(3), 2012, pp. 437-455.
[85] B. Köllner, S. Ruhle, and S. Süß, "The moderating role of message content in the formation of employee voice", German Journal of Human Resource Management, 33(1), 2018, pp. 56-75.

[86] D. Gould, "Using vignettes to collect data for nursing research studies: how valid are the findings?", Journal of Clinical Nursing, 5(4), 1996, pp. 207-212.

[87] J. Berg, M. Furrer, E. Harmon, U. Rani, and M.S. Silberman, "Digital labour platforms and the future of work: Towards decent work in the online world", International Labour Office, 2018.

[88] Tabachnick, B.G., and L.S. Fidell, Using Multivariate Statistics, 6th edn., Prentice Hall, 2012.

[89] S. Dow, A. Kulkarni, S. Klemmer, and B. Hartmann, "Shepherding the crowd yields better work", in Proceedings of the ACM 2012 conference on Computer Supported Cooperative Work, 2/11/2012 - 2/15/2012. 2012. ACM: New York, NY, pp. 1013-1022.

[90] V. Gupta, J.M. Fernandez-Crehuet, and T. Hanne, "Freelancers in the Software Development Process: A Systematic Mapping Study", Processes, 8(10), 2020, 1215, pp. 1-25.

[91] N. Galais, and K. Moser, "Organizational commitment and the well-being of temporary agency workers: A longitudinal study", Human Relations, 62(4), 2009, pp. 589-620.

[92] S. Suharto, and N. Hendri, "The impact of organizational commitment on job performance.", International Journal of Economics \& Business Administration, 7(2), 2019, pp. 189-206.

[93] M.H. Birnbaum, "How to show that 9>221: Collect judgments in a between-subjects design", Psychological Methods, 4(3), 1999, pp. 243-249. 